\title{
Alopécia Fibrosante Frontal associada a Líquen Plano Pigmentoso - Relato de 2 Casos
}

\author{
Paniagua DCl, Santos TS², Jeunon TSV³, Marques-Da-Costa JC4, Melo DF \\ 'Médica do Departamento de Dermatologia/Medical Doctor, Hospital Naval Marcílio Dias, Brasil \\ ${ }^{2}$ Médica do Departamento de Dermatologia/Medical Doctor, Hospital Naval Marcílio Dias, Brasil \\ ${ }^{3}$ Dermatologista e Dermatopatologista da Investigação em Dermatologia (ID)/Dermatologist, Patho-logist and Investigator in \\ Dermatology, Brasil \\ ${ }^{4}$ Médica Preceptora do Departamento de Dermatologia/Tutor in Dermatology, Hospital Naval Mar-cílio Dias, Brasil \\ ${ }^{5}$ Médico Preceptor do Departamento de Dermatologia/Tutor in Dermatology, Hospital Naval Marcí-lio Dias, Brasil
}

RESUMO - A alopecia fibrosante frontal, variante do líquen plano pilar, foi descrita por Kossard em 1994. Trata-se de uma alopecia cicatricial caracterizada por rarefação progressiva da linha de implantação fronto-temporal. A dermatoscopia tem grande importância no aumento da acurácia diagnóstica, sendo uma relevante ferramenta para distingui-la dos demais diagnósticos diferenciais. $O$ líquen plano pigmentoso, é outra variante do líquen plano, de ocorrência incomum, que ocorre principalmente em mulheres de fototipo III e IV entre a terceira e quarta décadas de vida. Neste artigo serão relatados dois casos da rara associação entre líquen plano pigmentoso e alopecia fibrosante frontal em duas pacientes femininas, de 59 e 76 anos, melanodérmicas, apresentando rarefação capilar marginal fronto-temporal e hiperpigmentação acastanhada da face, região cervical e tronco.

PALAVRAS-CHAVE - Alopecia; Líquen Plano.

\section{Frontal Fibrosing Alopecia with Lichen Planus Pigmentosus - Two Case Reports}

ABSTRACT - Frontal fibrosing alopecia, a variant of lichen planopilaris, was first described by Kossard in 1994 . It consists of a cicatricial alopecia characterized by progressive rarefaction of frontotemporal implantation line. Dermoscopy has a great importance to improve diagnosis accuracy and is a relevant tool to distinguish it from other differential diagnosis. Lichen planus pigmentosus is another variant of lichen planus, of uncommon occurrence, that specially affects women with phototypes III and IV, between third and fourth decades of life. In this article, two cases of the rare association of Lichen planus pigmentosus and frontal fibrosing alopecia are reported in two female patients, aged 59 and 76 years melanodermic women, presenting frontotemporal margin hair loss and brown hyperpigmentation on the face, neck and trunk.

KEY-WORDS - Alopecia; Lichen Planus.

\section{INTRODUÇÃO}

Este artigo se propõe a relatar dois casos raros e exuberantes da associação entre a alopecia fibrosante frontal (AFF) e o líquen plano pigmentoso (LPP) na face. Ambas as condições clínicas são variantes do líquen plano, têm etiologia desconhecida e predominam em mulheres. A dermatoscopia e a histopatologia auxiliam no diagnóstico, porém o manejo é difícil e as opções terapêuticas limitadas.

\section{RELATO DOS CASOS}

\section{Paciente 1}

Paciente do sexo feminino, 59 anos, fototipo V, saudável, menopausada há 10 anos. Queixa-se de perda dos cabelos da região frontal do couro cabeludo e queda progressiva dos supracílios há aproximadamente cinco anos, tendo feito maquiagem definitiva no local.

Ao exame, presença de rarefação capilar marginal
Correspondência: Thuany Silva Santos

E-mail:santosthuany@yahoo.com.br
Recebido/Received 5 Março /5 March 2016

Aceite/Accepted

8 Abril/8 April 2016 


\section{Dermatoscopia}

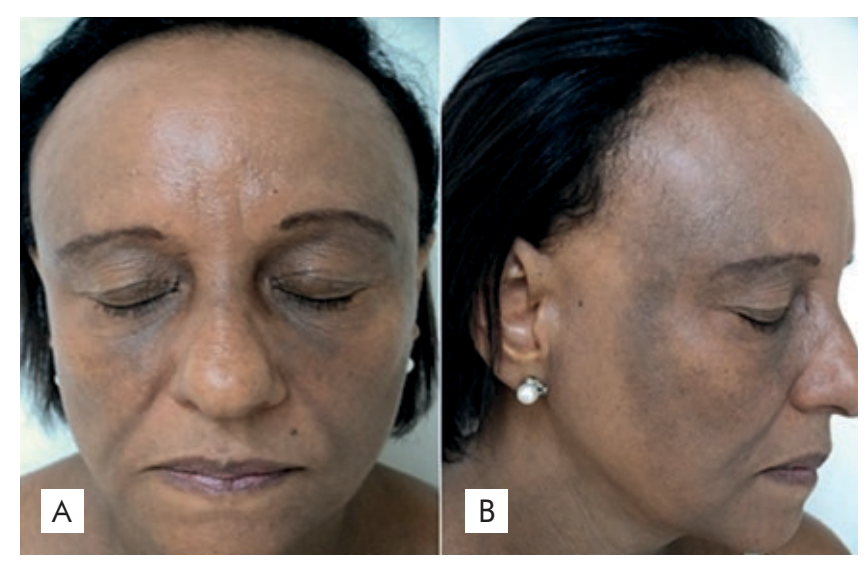

Figura 1 - Rarefação capilar marginal fronto-temporal com eritema e ceratose nos óstios foliculares da linha de implantação capilar.

fronto-temporal com eritema e ceratose nos óstios foliculares da linha de implantação capilar dessa região (Fig. 1). Apresenta ainda, hiperpigmentação acastanhada da face, região cervical e tronco, não responsiva ao tratamento tópico prévio com despigmentantes. Nega sintomas associados.

À dermatoscopia, notam-se pápulas foliculares com eritema perifolicular, escamas abraçando a haste capilar na altura da emergência dos óstios foliculares (padrão em estalagmite) no couro cabeludo (Fig.s. 2A-2B) e na face, presença de pseudorede acinzentada (Fig. 2C).

A investigação incluiu hemograma, TSH, anticorpos antitireoideus, anticorpos antinucleares e VDRL, todos normais e biópsia da face na região temporal. $\bigcirc$ estudo histopatológico evidenciou discreta infiltração inflamatória perivascular superficial constituída por linfócitos e numerosos melanófagos, associada à retificação dos cones epidérmicos, degeneração vacuolar da camada basal, esparsos queratinócitos em necrose individual e raros linfócitos em alinhamento com a junção dermoepidérmica. A camada granulosa e a camada córnea tinham aspecto habitual (Fig. 3). Esses achados são compatíveis com o diagnóstico de líquen plano pigmentoso.

Foi iniciada terapia intralesional com solução injetável de dipropionato de betametasona $5 \mathrm{mg} / \mathrm{mL}$ + fosfato dissodico de betametasona $2 \mathrm{mg} / \mathrm{mL}$ (sem dilvição) nas áreas de líquen plano pilar com intervalos de 4 semanas, solução de sulfato de minoxidil a $5 \% 2 x$ /dia nas sobrancelhas e aplicação tópica de creme de clobetasol 1x/dia na face por 30 dias seguida de pomada

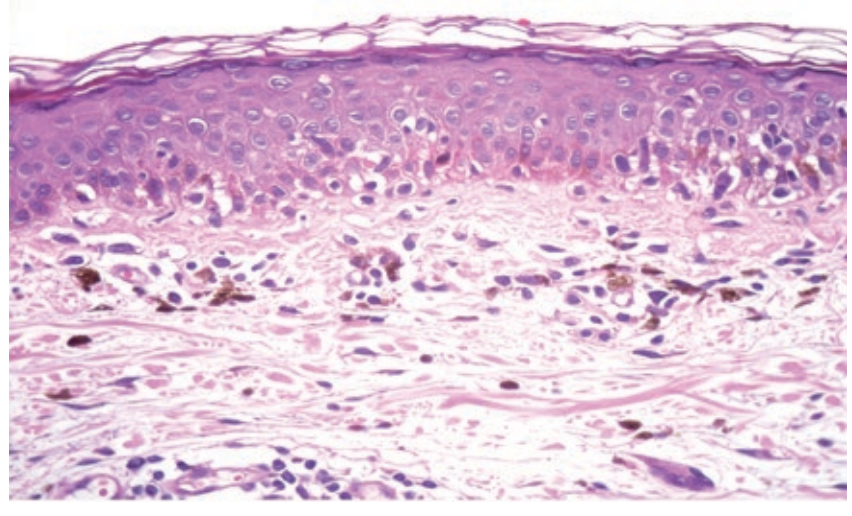

Figura 3 - Epiderme exibindo retificação dos cones, degeneração vacuolar da camada basal e esparsos queratinócitos em necrose individual, associada a numerosos melanófagos e escassa infiltração inflamatória da derme superficial (H\&E, 400 x).

de tacrolimus $0,1 \%$ por mais 30 dias. Após algumas semanas a paciente respondeu com discreta melhora do quadro.

\section{Paciente 2}

Paciente do sexo feminino, 76 anos, fototipo VI, menopausada há vinte anos, portadora de hipertensão arterial, diabetes mellitus tipo 2 e insuficiência renal crónica. Veio ao ambulatório queixando-se de queda de cabelo associada a prurido e manchas escuras no rosto há mais de 10 anos.

Ao exame, apresenta rarefação capilar difusa com retração da margem de implantação capilar, pápulas ceratósicas e eritema nos óstios foliculares da região frontotemporal (Fig. 4), rarefação bilateral dos supracilios e hiperpigmentação acastanhada difusa na face, pescoço e colo, não responsiva ao uso de diversos despigmentantes. À dermatoscopia, observamos pápulas foliculares com eritema perifolicular, escamas abraçando os folículos na altura da emergência dos óstios no couro cabeludo (Fig. 5A-5B) e pseudorede marrom homogénea com focos de hiperpigmentação castanho-acinzentadas na face (Fig. 5C).

A pesquisa laboratorial com análise do hemograma, dosagens de TSH, anticorpos antitireoideus, anticorpos antinucleares e VDRL não demonstrou alterações. A biópsia da região frontal, próxima do supracílio, evidenciou preservação das camadas córnea e granulosa, retificação dos cones

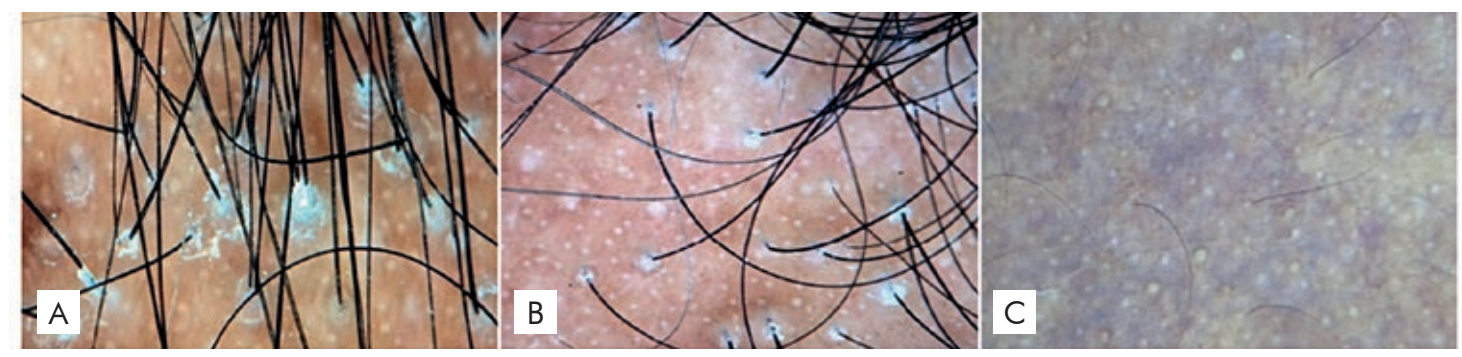

Figura 2 - A e B - Tricoscopia, C - Dermatoscopia. 


\section{Dermatoscopia}

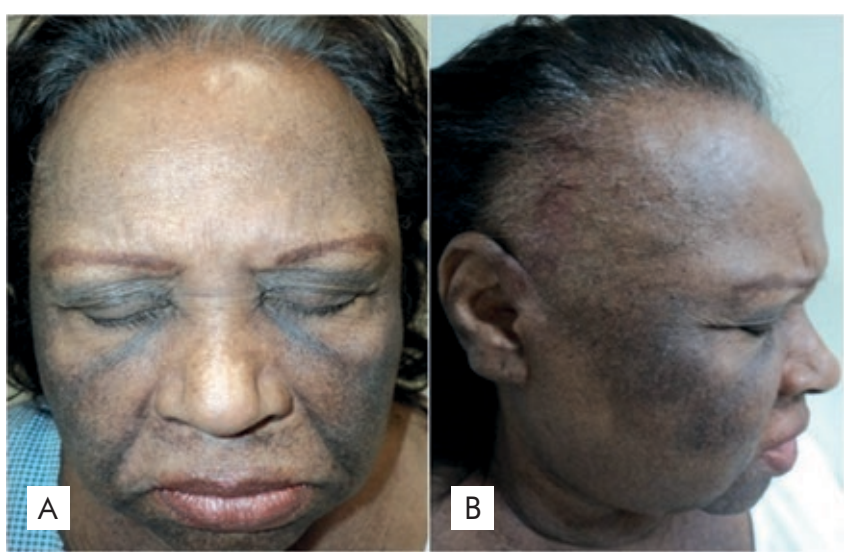

Figura 4 - Rarefação capilar difusa com retração da margem de implantação capilar e presença de pápulas ceratósicas e eritema nos óstios foliculares da região frontotemporal. de sulfato de minoxidil a $5 \% 2 x /$ dia nas sobrancelhas. Os despigmentantes foram suspensos e aplicação tópica de mometasona em creme $1 \mathrm{mg} / \mathrm{g}$ foi iniciada $1 \mathrm{x} /$ dia na face e no colo. Após 30 dias, a paciente referia melhora parcial do quadro, tanto do prurido, quanto da queda capilar e das manchas na face.

\section{DISCUSSÃO}

Alopecia fibrosante frontal (AFF) é uma alopecia cicatricial envolvendo couro cabeludo, sobrancelhas e outras áreas de pilosas, descrita pela primeira vez por Kossard como uma rarefação progressiva da linha frontotemporal.' Em sua maioria, os pacientes são mulheres na pós-menopausa e está muitas vezes associada com alopecia de tração. ${ }^{2,3} \mathrm{Na}$ última década, observou-se uma frequência crescente de casos de AFF. Geralmente tem ocorrência esporádica, mas tem sido relatada história familiar. ${ }^{4,5}$
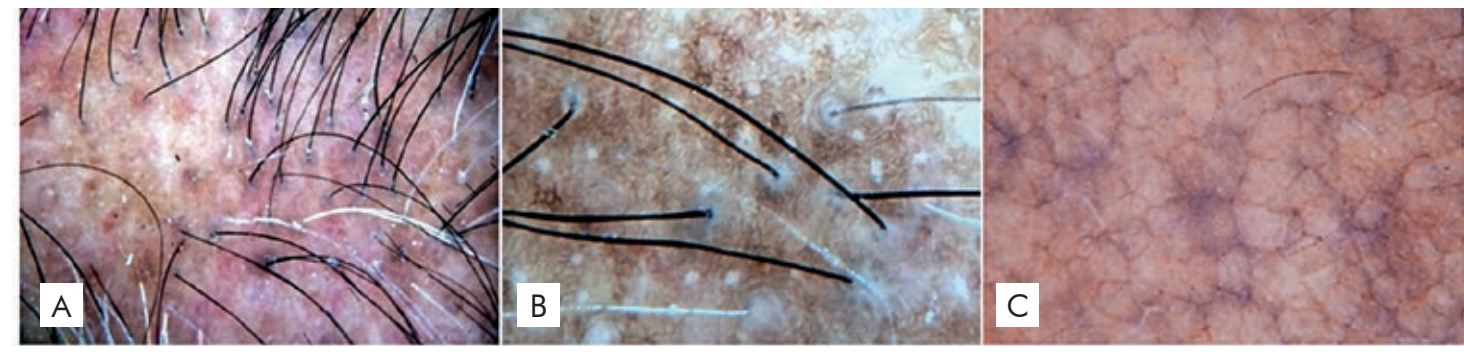

Figura 5 - A e B - Tricoscopia, C - Dermatoscopia.

epidérmicos, focos isolados de degeneração da camada basal, numerosos corpúsculos de Civatte e melanófagos na derme superficial, acompanhados de escassa infiltração inflamatória linfocitária perivascular (Fig. 6), achados compatíveis com o diagnóstico de líquen plano pigmentoso.

Foi iniciada terapia tópica com solução capilar de propionato de clobetasol $0,5 \mathrm{mg} / \mathrm{mL}$ no couro cabeludo e solução

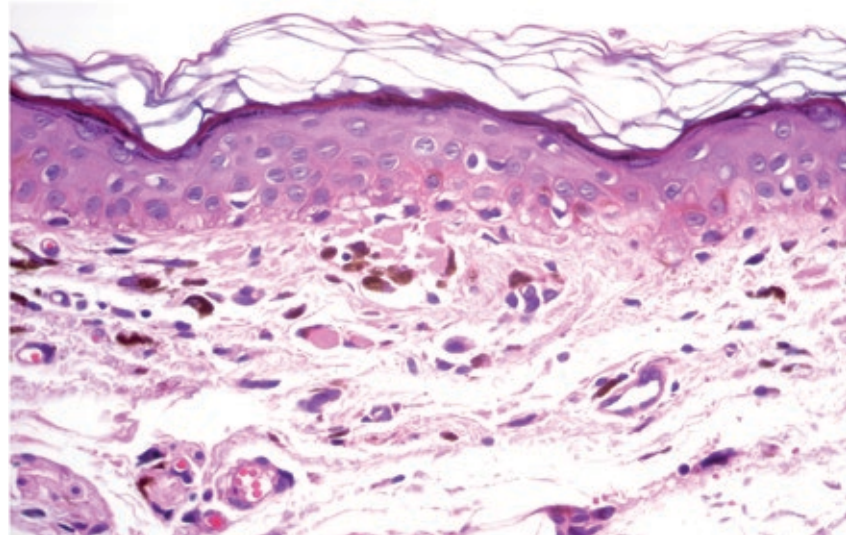

Figura $\mathbf{6}$ - Epiderme retificada, com degeneração vacuolar da camada basal. A derme apresenta numerosos corpúsculos de Civatte, melanófagos e escassa infiltração inflamatória linfocitária perivascular $(H \& E, 400 x)$
Trata-se de uma alopecia cicatricial que destrói os folículos pilosos da região fronto-temporal com frequente redução da pilificação das sobrancelhas e envolvimento esporádico dos cílios, axilas, regiões inguinais e membros, podendo preceder ou suceder do LPP. 6

A AFF é classificada atualmente como variante do líquen plano pilar, que é a forma mais comum de alopecia cicatricial primária em mulheres, com três variantes clínicas: o LPP clássico, alopecia frontal fibrosante e síndrome de Graham- Little. Contudo, é distinto do LPP clássico e da síndrome de Picardi-Lasseur-Graham-Little (LPP associado a pápulas hiperceratóticas foliculares em membros, tronco, axila, púbis e sobrancelha). $\mathrm{Na}$ AFF não se observam lesões de líquen plano em outros locais. ${ }^{7}$

As características histológicas da AFF e do LPP são semelhantes: infiltrado inflamatório linfocítico envolvendo o istmo e infundíbulo, queratinócitos apoptóticos na raiz da bainha externa, fibrose perifolicular e trato fibroso. ${ }^{3} \mathrm{O}$ infiltrado inflamatório na AFF predomina na parte superior do folículo piloso, ao passo que, no líquen plano, o infiltrado inflamatório não está limitado ao istmo e a fibrose perifolicular não é uma característica marcante como na AFF.8,9

A histopatologia a AFF caracteriza-se predominantemente pela presença de: prominente fibrose perifolicular; infiltrado linfocitico com padrão liquenóide ao redor dos folículos; e redução do número de folículos e substituição dos mesmos por tratos fibrosos. 


\section{Dermatoscopia}

A destruição do bulge (sítio das células germinativas) é a responsável pela alopecia permanente, característica da doença. Nota-se também degeneração hidrópica da bainha radicular externa (BRE) e preservação da epiderme interfolicular.

Sugere-se que a biopsia seja feita com dois punchs $4 \mathrm{~mm}$ e enviada para cortes transversais e longitudinais ${ }^{3}$. A imunofluoresencia da AFF é negativa e os achados histopatológicos são menos exuberantes que os do líquen plano pilar clássico.

A dermatoscopia pode ser uma ferramenta útil para o aumento da acuracia diagnóstica. Na AFF os pelos velos são tipicamente ausentes na linha de implantação do cabelo. 5,10 Os principais achados dermatoscópicos no couro cabeludo são: perda das aberturas dos óstios foliculares; eritema perifolicular; escamas perifoliculares aderidas a base das hastes capilares.

Aproximadamente $30 \%$ dos pacientes com AFF podem apresentar doença autoimune. $3 \mathrm{~A}$ investigação laboratorial inclui hemograma completo, hormonas sexuais, função tireoideia, anticorpos antinucleares, sorologia para hepatites e VDRL. ${ }^{9}$

Os principais diagnósticos diferenciais são: alopecias por tração, areata ofiásica inversa, androgenética, marginal cicatricial, senescente e lúpus eritematoso cutâneo crônico.

A AFF em geral é uma doença crónica progressiva, com tendência à estabilização do quadro e, embora existam muitas opções de tratamento (retinóides tópicos e sistémicos, esteróides tópicos ou intralesionais, hidroxicloroquina, minoxidil e finasterida) $)^{3,11}$ a resposta terapêutica é desanimadora. Vano-Galván et al. afirmam que, no seu estudo, as terapias mais eficazes foram finasterida e dutasterida orais seguidas por corticosteróides intralesionais. ${ }^{12}$

O líquen plano pigmentoso (LPP), por outro lado, é uma variante macular incomum do líquen plano. ${ }^{2}$ Apresenta-se clinicamente como máculas castanho-acinzentadas, ovais, nas áreas fotoexpostas da face, pescoço e áreas intertriginosas. Menos de 1/3 dos casos tem associação com alterações na mucosa ou com lesões típicas de líquen plano. Pode estar associado a prurido leve ou ardor.

A etiologia do LPP é desconhecida, porém acredita-se na participação de mecanismos imunológicos e sua histopatologia demonstra degeneração vacuolar da camada basal, infiltrado liquenóide variável e atrofia epidérmica, como no presente caso (Fig. 5A-5B).

São diagnósticos diferenciais possíveis melasma, dermatose cinzenta, hiperpigmentação induzida por drogas, hiperpigmentação pós-inflamatória, líquen plano actínico, dermatite de contato pigmentada (melanose de Riehl), ocronose exógena e eritema discrómico perstans.

A terapêutica do LPP é feita com fotoproteção, clareadores, corticóides tópicos ou orais com melhora variável. $\bigcirc$ uso de ciclosporina sistémica tem sido reservado para casos graves; luz intensa pulsada (LIP) é muito utilizada para o tratamento de algumas lesões pigmentadas com resultados promissores. ${ }^{2,3}$

A associação da AFF com LPP é pouco comum, estando descrita sobretudo em mulheres (95\%) e afrodescendentes $(91 \%),{ }^{2}$ o que é corroborado no nosso trabalho. Acredita-se que as duas condições sejam espectro da mesma doença.
Sabe-se que essas duas condições clínicas estabilizam com o tempo mas não está claro quando e como isso ocorre, havendo necessidade de estudos complementares para maiores esclarecimentos.

Conflitos de interesse: Os autores declaram não possuir conflitos de interesse. Suporte financeiro: $O$ presente trabalho não foi suportado por nenhum subsídio ou bolsa. Direito à privacidade e consentimento escrito: Os autores declaram que pediram consentimento ao doente para usar as imagens no artigo.

Conflicts of interest: The authors have no conflicts of interest to declare. Financing Support: This work has not received any contribution, grant or scholarship. Privacy policy and informed consent: The authors declare that the patient gave written informed consent for the use of its photos in this article.

\section{REFERÊNCIAS}

1. Rao R, Sarda A, Khanna R, Balachandran C. Coexistence of frontal fibrosing alopecia with lichen planus pigmentosus. Int J Dermatol. 2014; 53:622-4.

2. Dlova NC. Frontal fibrosing alopecia and lichen planus pigmentosus: is there a link? $\mathrm{Br} J$ Dermatol. 2013; 168:439-42.

3. MacDonald A, Clark C, Holmes S. Frontal fibrosing alopecia: a review of 60 cases. J Am Acad Dermatol. 2012; 67:955-61.

4. Donati A, Molina L, Doche I, Valente NS, Romiti R. Facial papules in frontal fibrosing alopecia: evidence of vellus follicle involvement. American academy of pediatrics. Arch Dermatol. 2011 ; 147:1424-7.

5. Rubegni $P$, Mandato F, Fimiani M. Frontal fibrosing alopecia: Role of dermoscopy in differential diagnosis. Case Rep Dermatol. 2010; 2:404-5.

6. Mirmirani P, Karnik P. Lichen plano pilari streated with a peroxisome proliferator-activated receptor gammaagonist. Arch Dermatol. 2009; 145:1363-6.

7. Mulinari Brenner F, Rosas FM, Sato MS, Werner B. Frontal fibrosing alopecia: report of six cases. An. Bras.Dermatol. 2007; 82:439-44.

8. Moreno Ramírez D, Camacho Martínez F. Frontal fibrosing alopecia: a survey in 16 patients. J Eur Acad Dermatol Venereol. 2005; 19:700-5.

9. Tosti A, Piraccini BM, lorizzo M, Misciali C. Frontal fibrosing alopecia in postmenopausa women.J Am Acad Dermatol. 2005; 52:55-60.

10. Yin NC, Tosti A. A systematic approach to Afro-textured hair disorders: dermatoscopy and when to biopsy. Dermatol Clin. 2014; 32:145-51.

11. Berliner JG, McCalmont TH, Price VH, Berger TG. Frontal fibrosing alopecia and lichen planus pigmentosus. J Am Acad Dermatol. 2014; $71:$ e26-7.

12. Vañó-Galván S, Molina-Ruiz AM, Serrano-Falcón C, Arias-Santiago S, Rodrigues-Barata AR, Garnacho-Saucedo G, et al. Frontal fibrosing alopecia: a multicenter review of 355 patients. J Am Acad Dermatol. 2014; 70:670-8. 